\title{
Progressive Lightning: A New Stereoscope
}

\author{
By Dr. C. V. Boys, F.R.s.
}

$\mathrm{A}$ MOST interesting and illuminating paper on the subject of progressive lightning by Dr. E. C. Halliday ${ }^{1}$ describes the results of successful experiments made by himself in South Africa at the instance of Dr. B. F. J. Schonland with an instrument similar to that which I made in the year 1900. As I gave the first description of my instrument in NATURE ${ }^{2}$ and have contributed other articles on this subject exclusively to these columns $^{3}$ I should be glad to use the same medium for some observations on Dr. Halliday's paper and at the same time describe a new stereoscope designed to examine these photographs without cutting them and also to obtain the physiological advantage of contrast due to reversed stereoscopic effect.

The method of examination of the progress of a lightning flash depends on the use of two images of the flash carried over a photographic plate at a high speed in opposite directions, or of a photographic plate or film carried past two images of the flash in opposite directions. If, then, any part of the flash is subsequent to any other part to an extent visible on the images, these will be distorted in opposite directions and a comparison of the images will give all the information available.

$I$ had the temerity to invent this method of attack in the year 1900 and within a week I had constructed myself an instrument for the purpose. I carried this instrument about, chasing lightning without success, until in the year 1928 I caught up with a flash in America. This photograph, reproduced in NATURE of September 1, 1928, clearly demonstrated that my idea that lightning might occupy some appreciable time while blazing its trail was correct, and Dr. Halliday's photographs confirm this as well as my prediction that an immediately subsequent flash along the same path would be so quick that no aberration would be apparent in the images.

Dr. Halliday's instrument is somewhat less powerful than mine of 1900 but it appears to be ample for the purpose of getting measurable aberration. These instruments are far less powerful than that which Mr. Loomis has at Tuxedo Park, New York, or than the one of similar design which Prof. Adolf Matthias informed me in 1929 that he was going to make. I have not heard whether this instrument ever was made or, if so, whether he has obtained any results. Mr. Loomis and Prof. R. W. Wood have been hunting lightning for two summers with the instrument illustrated diagrammatically in NATURE of July 13, 1929, but so far without success. This perhaps is not surprising, since it was twenty-eight years before I caught a single flash. Thanks to the courtesy of Mr. Goodlet of the Metropolitan-Vickers Electrical Co., Ltd., I was able to tost this instrument on their high tension sparks before sending it out to
Mr. Loomis. There was never any question of observing progressive development of the 5-ft. spark, but the photographs were so perfect, more especially when Tesla sparks were examined, that the good behaviour of the instrument was assured beforehand. This instrument was constructed by Messrs. Ross, Ltd., at their Clapham works and it is as perfect as such a thing can be. My only fear was that perhaps lightning might be too slow for corresponding pairs of images to be recognisable as being of the same flash.

My method of investigating the progress of a lightning flash has also attracted attention in Western Australia and in Western America, but not apparently in England. I need scarcely say that it has been a source of great satisfaction to me to see Dr. Halliday's paper on his work in South Africa. He sent me an advance draft last autumn but I felt unable to make any reference to it until it had appeared. For this reason I have held back for six months the description herewith of a new stereoscope for observing the progressive character of a flash. That is, unlike any other stereoscope, the instrument indicates time or delay and not solid form.

Coming now to Dr. Halliday's photographs, my object just now is to consider not exactly how his flashes behaved, where they started or how long they took to cover their course, but in the first place to refer to a new point which he has made. It is a little curious that in my American flash, the two images happened to be one above the other and so to be moving horizontally at the time of the flash. Any aberration would result in a bending or a tilting in opposite directions of the two images of the flash, which was nearly vertical. In Dr. Halliday's photographs, on the other hand, the two images happened to be at about the same level and so were moving vertically. According to the aberration that I had contemplated, this would lead to a lengthening of one image and a shortening of the other, and in that case the stereoscope would indicate exactly the same time relief as with the lenses in the other position. Dr. Halliday, however, introduces a new idea. He imagines a flash starting at the cloud and moving downwards. Then he says that the starting point of the flash will remain luminous all the time that is occupied by the flash in travelling to its other end. I suppose he means that electric current would be pouring in at the starting point all this time and keeping it alight. In such case, with the images moving vertically, one image moving towards the cloud's image would, he says, be of its proper length, for the image of the upper end of the flash would be carried towards the cloud exactly to the same extent that the image of the lower end would be carried towards that of the upper end. On the other hand, the 
other image would be shortened exactly as I have always contemplated. Unfortunately, Dr. Halliday had such wide angle lenses, $F 2 \cdot 7$, that he had far too much light and his photographs are much spoilt by glare. In my original camera, I used about $F 8$ and with about one-eleventh of the light obtained clear images. I expect $F 11$ or even $F 16$ would give sufficient illumination for the main flash.

Now if my conclusion that the American flash began in the middle is correct, it is difficult to see where the reservoir was to keep up the current. Also, as a matter of fact, the images do not show any broadening at the centre or anywhere. Had current been poured in all the time that the flash was travelling, the image where it started would have been wider than where it finished. According to my view, this lightning flash had some similarity with the flames over a coal fire. Anyone looking at these would believe that the flames were continuous, but if he will look through a moving slit so as to catch a rapid succession of momentary views, he will see that the flame is broken up into a number of elements, and it is the travelling of these upwards in succession that gives the appearance of one long continuous flame. A cinematograph view would be of interest. Similarly, my photograph with images of the same width throughout but slightly different in shape indicates to me that the bright part of the flash travelled from the starting point to the terminal points and that the starting point was not luminous all the time that the flash lasted.

It may well be that no two flashes behave alike and $I$ am not making any point of this difference of view. The real interest to me as an experimentalist is the evidence that the method is capable of ascertaining the facts whatever these facts may be, rather than in worrying over any initial differences in outlook. It seems to me to be far more important to find out what lightning really does than to trouble about ideas of what it ought to do.

It might be worth while to use four lenses equally spaced round a circle so as to obtain for every flash confirmatory evidence of its progress.

\section{The New Stereoscope}

Fig. 1 in NATURE of September 1, 1928, was obtained by cutting a print in two and rotating each half through a right angle. To ensure this, a straight line had been ruled on the original negative close to and parallel to the line joining the two images, and the two half prints were turned through a right angle until these became vertical. I have found that if I cut up two prints and put two images on each side close together but reversed, that is each the same way up but so that one pair have their sharp edges adjacent while the other have their fading away edges adjacent, the stereoscopic effect of the two pairs is reversed and the contrast makes the effect the more conspicuous.
It is desirable, however, to be able to examine stereoscopically a print without cutting it, and if the contrast due to reversal can be obtained so much the better. This can be done in the following way. Make up a stereoscope by cutting a double convex lens in half and mounting these with their cut diameters away from one another. Then make two isosceles right angle prisms of glass truncated so that the right angle is absent and only the useful part near the hypotheneuse remains. The result is a rectangular prism with inclined ends and the rectangle is best a square. Fig. 1 shows how I have slit an ordinary reflecting prism into three pieces, utilising the two bottom parts. Mount these in two tubes so that they act as reversing mirrors when anything is seen through them. Mount these tubes above the two half lenses at eye distance and arrange that each tube can be turned on its own axis through a right angle, limited at each extremity by screw stops. If now the prisms in these tubes are so placed and moved that all their corresponding edges are always

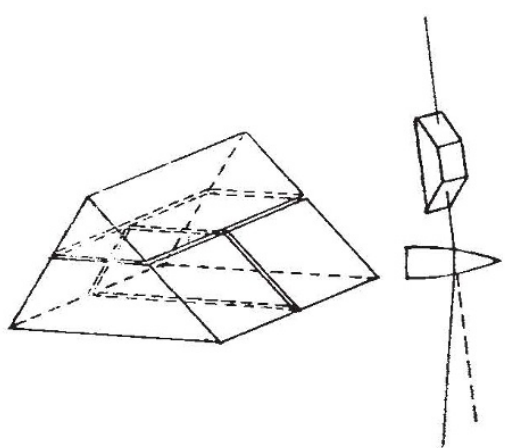

IIG. 1

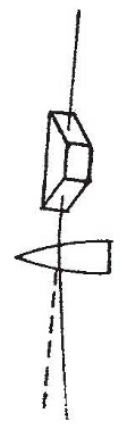

FIQ. 2 parallel except for the slight convergence, and the square prisms are placed corner-wise so as to turn the images round in the same direction through a right angle as seen by the eyes, these images will then show stereoscopic relief if they are different in form due to aberration. If, then, the tubes are suddenly turned each through a right angle, the images will both be inverted in position and the stereoscopic appearance will be reversed, thus by contrast accentuating the stereoscopic effect. Fig. 2 shows the optical parts only in one position.

It may be worth while to say that the shorter the image of a flash is the greater is the stereoscopic impression, because the apparently further and nearer parts, that is, actually the earlier and later, are more closely adjacent on the plate. For this reason it is well to photograph lightning which is not nearer than four or five miles. Short focus lenses also have an advantage in this respect over lenses of longer focus. I did succeed in obtaining with my original instrument quite a spectacular photograph of lightning not a quarter of a mile away and two others on the same plate within a mile. Unfortunately, they are all so much to one side that part of one image of each is off the plate. These were obtained in the night 
of August 29, 1930, when a terrific thunderstorm of blinding flashes came to St. Marybourne from Winchester, but no flashes were clear of obstruction by rain until they were close up.

Dr. Halliday has made no reference to the stereoscopic method of examination. In his photographs the process of measurement with a micrometer microscope is easy as the aberration is longitudinal. In my photograph where this is transverse, $I$ found measurement with Mr. Loomis's fine micrometer microscope too tedious and the stereoscopic method much more convenient.

I should like in conclusion again to appeal to lightning observers suitably placed to initiate a lightning flash with a rocket. I gave some indica- tion of what to do in my article in Nature of November 20,1926 , but if anyone should care to consider this more closely, I would recommend the construction of a 'towering rocket' of suitable proportions which should have great persuasive power. Long ago I made a $1 \mathrm{lb}$. rocket to carry a $6 \mathrm{oz}$. rocket in its head and this again to carry a $2 \mathrm{oz}$. rocket which, of course, had the usual stars. The conducting trail which such a combination creates in a very short time and up to an immense height, should initiate a flash almost with certainty if a new flash should be becoming due

${ }^{1}$ Phil. Mag., Feb. 1933.

2 NATURE, 118, 749, Nov. 20, 1926.

${ }^{3}$ NATURE, 122, 310, Scpt. 1, $1928 ; 124,54$, July 13, 1929; and 127, 425, March 14, 1931.

\section{Researches on Gaseous Combustion* \\ By Prof. W. A. Bone, f.r.s.}

\section{Hydrocarbon Combustion}

$\mathrm{I}^{\mathrm{N}}$ reviewing, in my recent Bakerian lecture, the principal researches upon the combustion of hydrocarbons, the importance was stressed of a balanced judgment embracing the whole range of conditions from slow combustion through flames and explosions right up to detonation. For it is only by taking all conditions into account comprehensively that a true view of the subject can be gained.

It was shown how the hydroxylation theory is capable of expressing the principal facts of both the slow and explosive combustion of gaseous hydrocarbons. For although the conditions prevailing in flames are obviously much more complex than those of slow combustion, the main course of the chemical changes concerned therein may be satisfactorily interpreted on the supposition that the result of the initial encounters between hydrocarbon and oxygen is the same in both, namely, the formation of an 'oxygenated' which (except with acetylene) is a 'hydroxylated' molecule. Undoubtedly, at the higher temperatures of flames, secondary thermal decompositions occur and play a more conspicuous rôle than in slow combustion; but there are the strongest experimental grounds for believing that they do not precede the onslaught of the oxygen upon the hydrocarbon, but arise in consequence thereof. In particular, all the evidence is quite decisive against such views as that of a 'preferential' combustion, whether of hydrogen or carbon, or that in an explosion flame the hydrocarbon molecule is primarily resolved into its elements before being burnt.

Experiments were made showing that the affinities of hydrocarbons so greatly exceed those of either hydrogen or carbonic oxide for oxygen that in explosions of hydrocarbon-hydrogen (or carbonic oxide)-oxygen media deficient in oxygen the hydrocarbon is burnt, as it were, preferentially. Perhaps the most striking experiment of all consisted in exploding a mixture of 25 per cent of acetylene and 75 per cent of electrolytic gas in

- Substance of the Bakerian Lecture (Proc. Roy. Soc., A, 137, 243-274) and papers read afterwards at the Royal Society on Nov. 10, 1932 (ibid., A, 187, $57-83$ ). a stout glass bulb at a pressure of (say) $500 \mathrm{~mm}$. (higher could be used but for the danger of shattering the vessel). A sharp bluish flame filled the vessel, but neither carbon separated nor did any steam condense on cooling, the products consisting of carbonic oxide and hydrogen with traces only of carbonic anhydride or of methane. in accordance with the equation :

$$
\mathrm{C}_{2} \mathrm{H}_{2}+\mathrm{O}_{2}+2 \mathrm{H}_{2}=2 \mathrm{CO}+3 \mathrm{H}_{2} \text {. }
$$

A similar result was also obtained on exploding a mixture of equal volumes of ethylene, hydrogen and oxygen, thus :-

$$
\mathrm{C}_{2} \mathrm{H}_{4}+\mathrm{O}_{2}+\mathrm{H}_{2}=2 \mathrm{CO}+3 \mathrm{H}_{2} \text {. }
$$

Another most arresting and significant feature of the evidence, also illustrated experimentally, was that whereas all the hydrocarbons of the $\mathrm{C}_{n} \mathrm{H}_{2 n}$ series, that is, ethylene, propylene, trimethylene and butylene, on explosion with a $\mathrm{C}_{n} \mathrm{H}_{2 n}+{ }_{2}^{n} \mathrm{O}_{2}$ proportion of oxygen always yield substantially carbonic oxide and hydrogen only, as though there had been a preferential burning of their carbon, thus:

$$
\mathrm{C}_{n} \mathrm{H}_{2 n}+{ }_{2}^{n} \mathrm{O}_{2}=n \mathrm{CO}+n \mathrm{H}_{2} \text {, }
$$

explosions of corresponding members of the $\mathrm{C}_{n} \mathrm{H}_{2 n+2}$ or paraffin series (that is, ethane, propane or butane) with oxygen in the $\mathrm{C}_{n} \mathrm{H}_{2 n+2}+\frac{n}{2} \mathrm{O}_{2}$ proportion, all result in dense clouds of carkon, steam, methane and oxides of carbon. This striking difference between the behaviours of corresponding paraffins and olefines accords well with the hydroxylation theory.

Moreover, in regard to olefine explosions, another significant fact is that, so soon as the proportion of oxygen in the medium is progressively reduced below the $\mathrm{C}_{n} \mathrm{H}_{2 n}+{ }_{2}^{n} \mathrm{O}_{2}$ proportion, steam (as well as carbon) begins to appear in the products and relatively increases in amount as the oxygen content diminishes. This was demonstrated by exploding a $3 \mathrm{C}_{2} \mathrm{H}_{4}+2 \mathrm{O}_{2}$ mixture and it points unmistakably to the initial formation of 'hydroxy. lated' molecules in such explosions.

In recent years, however, the suggestion has been made that the initial association of hydro- 